\title{
Ultrafast Laser Processing of Glass
}

\author{
Kazuyoshi ITOH \\ Science Technology Entrepreneurship Laboratory, Office for University-Industry Collaboration, \\ Osaka University \\ E-mail: itoh@uic.osaka-u.ac.jp
}

\begin{abstract}
The emerging fabrication techniques for transparent materials such as glass and crystal that use focused ultra-short laser pulses will be reviewed from the viewpoint of functionalities of the products to be integrated into a future photonic system. The devices include waveguide and waveguide couplers, mirrors and lenses, filters and holograms. The future photonic system may consist of many functional subsystems which are connected through transparent gates or windows. Welding technique may play an important role for this system integration. The welding technique for transparent materials using ultra-short laser pulses will also be reviewed along with applications to hermetic sealing. The author presents experimental results on the temperature dynamics. The author will also discuss on an idea connecting the relaxation of internal stress and the high index change and a role of the void and its motion in the fabrication process. The bright near future and challenges of the glass processing techniques with ultrafast lasers will briefly be touched on.
\end{abstract}

DOI: 10.2961/ilmn.2014.03.0001

Keywords: laser processing, fabrication, optical device, welding, joining, index change, glass

\section{Introduction}

Glass is attractive industrial material because of its clear and wide-band transparency, chemical stability and wide varieties of ingredients. Although its surface can readily be toughened to some extent, the only week point is its brittleness. Focused ultrafast laser pulses can cause nonlinear absorption effects in glass, allowing us to conduct microprocessing inside of such materials. This technique has opened various applications such as optical memory [1, 2], fabrication of photonic devices [3], and welding of glass [4]. In this paper, we review mainly the development of novel techniques of ultrafast laser microprocessing and microwelding [5-7]. It is noted here that microprocessing and microwelding techniques are closely related for the future photonic system integration. The future photonic system may consist of many functional subsystems which are connected with transparent gates or windows. The microwelding technique may play an important role for this system integration. It is also noted that microwelding of glass excludes the use of adhesives or intermediate layers that can contaminate the surroundings and can be carried out substantially in room temperature. Thus, delicate devices such as the semiconductor light sources or sensors, MEMS devices and even solar cells can readily be integrated or hermetically sealed.

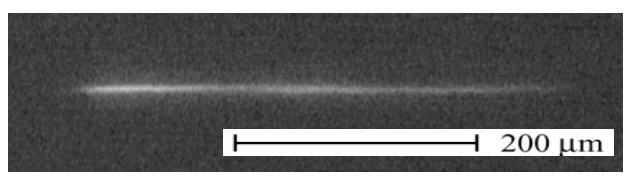

Fig. 1. Filamentation in silica glass.

\section{Laser Processing using Filamentation [8]}

The nonlinear propagation of an intense ultrafast laser pulse through a material can result in dynamic changes to its temporal, spatial, and spectral properties. Self-guiding of the beam created from laser pulses, which is also called filamentation, occurs as a consequence of dynamic balance between Kerr self-focusing and defocusing effects in the electron plasma generated through the nonlinear ionization process. Figure 1 shows a photograph of filamentation during the laser processing. Laser pulses with the $130-\mathrm{fs}$ duration and $1.0-\mu \mathrm{J}$ energy were focused from the left into silica glass with a single lens of numerical aperture (NA), 0.01. The picture is taken without illumination. A filamentary index change is inscribed at the place of the filamentation. Applications of filamentary propagation include the self-compression of the filamentation, lightning control, harmonic generation, terahertz radiation, atmospheric analysis, remote sensing of molecules, and supercontinuum generation [9-10]. Ultrafast laser filamentation is readily observed in transparent solids and liquids. The filamentation in glass that has typically a two-micron diameter and ranges from a few to a few hundreds of microns can be used for fabrication of three-dimensional photonic devices in glass and the welding/joining glass and other materials. In this paper we review applications of filamentation in glass to microprocessing and microwelding. The reader interested in details is referred to ref. [8].

\section{Laser Processing of Photonic Devices}

If you scan the filament in glass along the direction normal to the optical axis with an appropriate speed, you can inscribe a sheet of index change in glass. If you repeat this process, you can form layers of index change, a Bragg grating. Figure 2 shows optical micrographs of the fabricated gratings. Views from three different angles are shown [11]. A Bragg grating with a pitch of $5 \mu \mathrm{m}$ is formed. The pulse energy and the NA of the focusing lens 
were set to $1.0 \mu \mathrm{J}$ and 0.1 , respectively. The diffraction efficiency was $>70 \%$ for a He-Ne laser beam with TE polarization when the pitch was $3 \mu \mathrm{m}$.
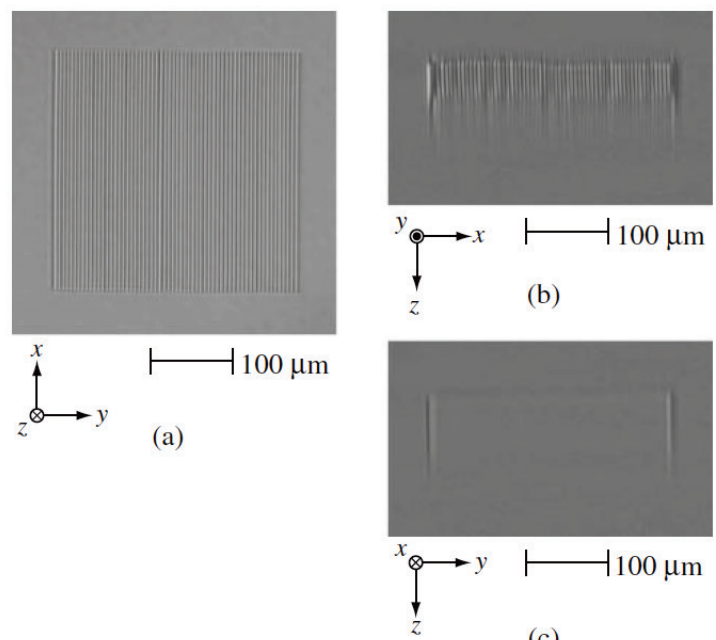

(c)

Fig. 2. Optical images of the fabricated Bragg gratings with the period of $5 \mu \mathrm{m}$. The fabrication energy was $1.0 \mu \mathrm{J}$ and the translation speed of the filament was $1 \mu \mathrm{m} / \mathrm{s}$. (a) Top view. (b) Side view (xz-plane). (c) Side view (yz-plane).

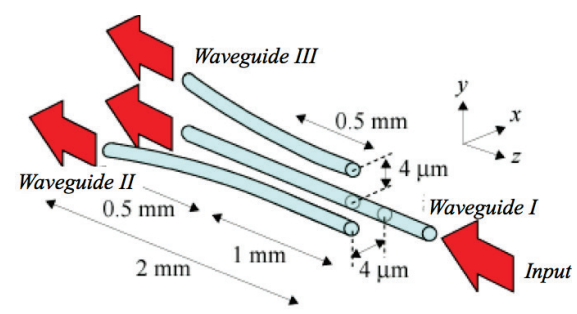

(a)

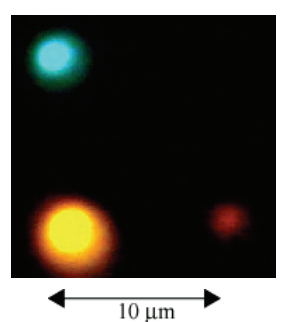

(b)

Fig. 3. (a) Design of a four-level phase-type diffractive lens. (b) Side view and (c) top view of a fabricated four level phase-type diffractive lens. (d) Focal spot when a $\mathrm{He}-\mathrm{Ne}$ laser beam $(632.8 \mathrm{~nm})$ was incident upon the lens.

Figure 3 shows a 3D wavelength division coupler fabricated in fused silica [12]. A $1-\mathrm{kHz}$ train of $130-\mathrm{fs}$ pulses at a wavelength of $800 \mathrm{~nm}$ was focused by an objective lens with a NA of 0.3 . When the pulse energy was $0.68 \mu \mathrm{J}$, filamentary propagation with a length of 40 $\mu \mathrm{m}$ was observed. Waveguides were formed by translating the sample position in the axial direction with a velocity of $0.2 \mu \mathrm{m} / \mathrm{s}$. Curved waveguides can be fabricated by translating the lateral position of the focus simultaneously with the axial position. By launching white light at the input of one of the waveguides, we observed different colors of light appeared at the different outputs as shown in Fig. 1(b).

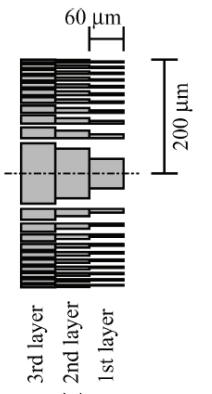

(a)

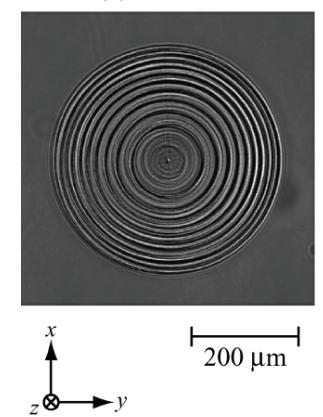

(c)

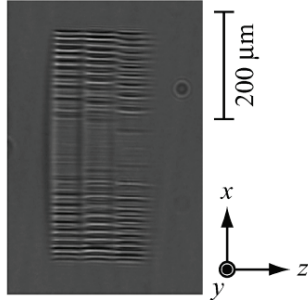

(b)

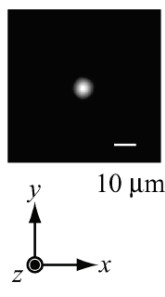

(d)
Fig. 4. (a) Design of a four-level phase-type diffractive lens. (b) Side view and (c) top view of a fabricated four level phase-type diffractive lens. (d) Focal spot when a $\mathrm{He}-\mathrm{Ne}$ laser beam $(632.8 \mathrm{~nm})$ was incident upon the lens.

Fiure 4 shows the result of fabrication experiment of four-level diffractive lens[13]. The design of the four-level lens is shown in Fig. 3(a). Each level of the lens consisted of a single layer. In the first layer a refractive-index change was induced in the retardation regions that satisfied $3 \pi / 2<\varphi(\mathrm{r})<2 \pi$. In the second layer a refractive-index change was induced in the retardation regions that satisfied $\pi<\varphi(\mathrm{r})<3 \pi / 2$. In the third layer a refractive-index change was induced in the retardation regions that satisfied $\pi / 2<\varphi(\mathrm{r})<\pi$. The thickness of each layer was designed to be $60-\mu \mathrm{m}$ because a $60-\mu \mathrm{m}$-thick region of refractive-index change gave a phase retardation of approximately $\pi / 2$. Side and top views of this lens are shown in Fig. 3 (b) and (c). The efficiency at the primary focal point was $56.9 \%$. Although this efficiency was higher than that of the two-level lens, this result was lower than the theoretical value. The reason for the discrepancy may be disagreement between the induced and the designed phase retardation. The measured profile of a focal spot is shown in Fig. 3(d); the resolution of the lens was $9.9 \mu \mathrm{m}$.

Many waveguide devices, optical elements such as volume and Dammann gratings, hologram and beam shaper are fabricated. The interested reader is referred to ref. [8]. 


\section{Laser Welding of Glass and Metal}

In order to integrate micro-photonic devices, it is important to assemble several micro-devices. Here we introduce the technique of ultrafast laser welding, which allows us to weld transparent glass materials [4]. Figure 5 shows a schematic of welding procedure. Ultrafast laser pulses are focused to the interface of transparent materials to be joined to cause nonlinear absorption. As a result, the optical energy is deposited to the material only around the focal point, leading to the generation of molten pool, which connects the gap between the samples. The molten pool is subsequently cooled down, forming a welded region. By translating the sample with respect to the focal spot, the two pieces of samples were joined in a point-by-point manner, and we can form welded regions with an arbitrary shape. Importantly, the selection of the NA of focusing lens allows one to adjust the length of the molten pool formed by the filamentation effect. If we use a laser with high repetition rate and the time interval between pulses is shorter than the cooling constant of the material, the heat near the focal area is accumulated and the situation becomes different [14].

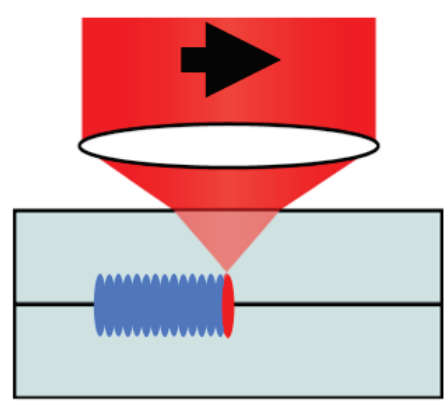

Fig. 5. Welding Procedure

An important advantage of this technique is that we can join dissimilar materials with a different thermal expansion coefficient. We consider that this advantage originates from the fact that the molten pool is as small as several micrometers and almost entire sample is kept at the room temperature, suppressing the thermal stress in a macroscopic scale. Indeed, we reported the successful joining between fused silica and borosilicate glass substrates [15] and that between BK7 and copper substrates [16].

The technique of laser micro welding can be used for hermetic sealing of ceramic and metal packages. Figure 6 shows the trial of hermetic sealing of ceramic package (92\% Alumina) and Schott D263 glass. A helium leakage test has been carried out on the sealed ceramic package. Even after the thermal cycle with temperatures of 125 and $-55^{\circ} \mathrm{C}$ was repeatedly applied 100 times each for 30 minutes, the hermeticity exhibited no significant degradation. This result proved that ultrafast laser micro-welding allows reliable hermetic sealing. [17].

\section{Temperature dynamics in glass fabrication}

We investigated the temperature dynamics during ultrafast laser microprocessing of glass by using time-resolved micro-Raman spectroscopy. We reported the spatial and temporal heat diffusion around the focal volume caused by irradiation of low-repetition rate femtosecond pulses [18]. On the other hand, highrepetition ultrafast laser pulses can induce smooth modifications at a high speed [14]. Temperature dynamics related to the high-repetition ultrafast laser pulses is quite important because the processed area is strongly influenced by the heat accumulation effect. We measured the temperature dynamics inside glass by time-resolved micro-Raman spectroscopy during high-repetition-rate femtosecond laser microprocessing [19].

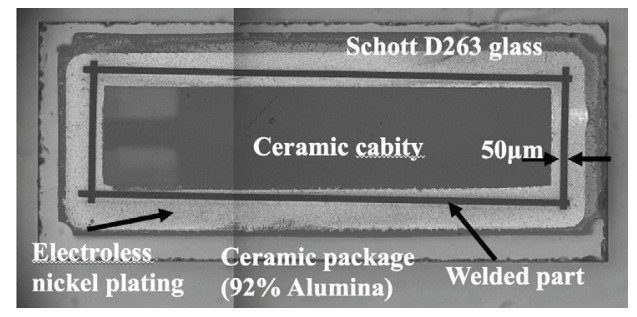

(a)

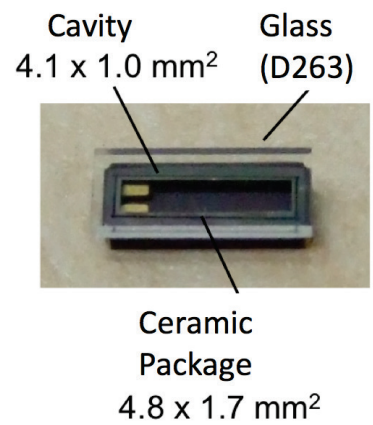

(b)

Fig. 6 Hermetically sealed sample of a ceramic package. (a) a magnified micrograph, (b) an outer appearance.

The experimental system is shown in Fig. 7. We used an amplified Ytterbium fiber laser system that was producing $1-\mathrm{MHz}$ and $600-\mathrm{fs}$ pulses at a wavelength of $1044 \mathrm{~nm}$ for fabrication. For Raman pump, Nd:YAG laser was used to generate 10-ns pulses at a wavelength of $532 \mathrm{~nm}$ and a repetition rate of $10 \mathrm{kHz}$. Their pulse energies were 1.1 and $0.3 \mu \mathrm{J}$, respectively. These pulses were electrically synchronized and then focused inside a sample $200-\mu \mathrm{m}$ below the surface by a $\times 20$ microscope objective lens with a numerical aperture of 0.46 . We used a plate of borofloat glass (Schott B33). A computer- controlled xy-stage was used for raster scanning of the sample at a velocity of 1 $\mathrm{mm} / \mathrm{s}$. The back-scattered Stokes and anti-Stokes Raman signals from the focus passed through a confocal pinhole were detected by a gated ICCD camera, whose gating time was set to $10 \mathrm{~ns}$. The number of accumulation times of the time-gated Raman signal was 20000. The temperature was calculated from the ratio of the Stokes to anti-Stokes Raman signals [19]. In order to measure spatial distribution of temperature, the focal position of the processing pulse was scanned perpendicular to the scan direction of the sample. 

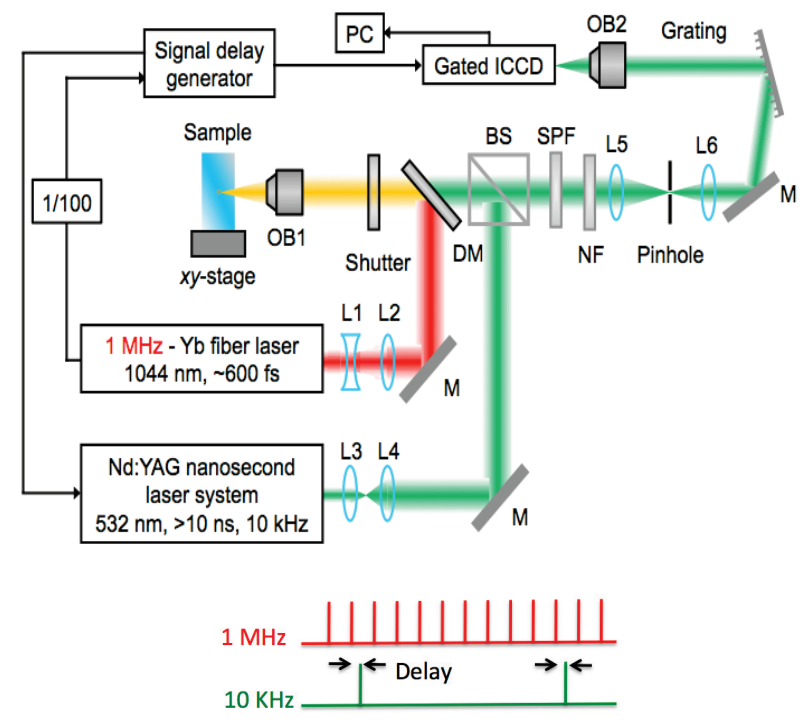

Fig. 7. Time-resolved micro-Raman measurement system for high-rep.-rate ultrafast laser microprocessing

Figure 8 shows the results of the Raman temperature measurement [19]. Within the range of $\pm 3 \mu \mathrm{m}$ from the focus of the processing pulse, it was difficult to measure temperature due to the onset of large background signals. Nevertheless, we were able to measure the temperature at the periphery of the focus of processing pulses. As shown in Fig. 8, we confirmed the decrease of the temperature depending on the position from the focus as expected. Please note that the spatial distribution is different from the Gaussian profile, which is expected for single-shot irradiation and that the estimated temperature at the focal area remains above $2500 \mathrm{~K}$ even $500 \mathrm{~ns}$ after the laser shot.

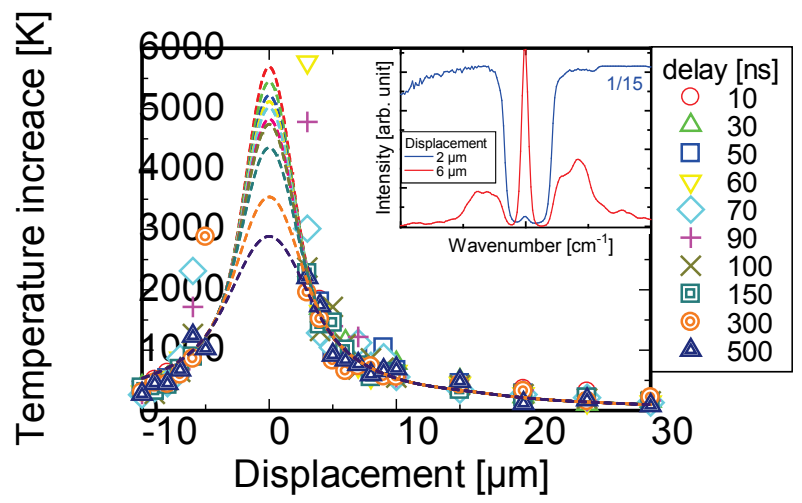

Fig. 8. Results of time-resolved micro-Raman temperature measurement during high-rep.-rate ultrafast laser micro-processing.

\section{Void formation, residual stress and index change}

High index increase is important for the compact photonic devices with the small minimum radius of curvature. The index change is considered as the results of densification of glass [20] and/or microexplosion [6] and usually accompanies residual stress. Figure 9 visualizes the internal stress after the raster-scanning modification experiment without void formation [21]. The central dark (blue) area is the processed area. It is shown that large stress is accumulated during the laser scanning. Figure 9 (b) indicates the stress distribution along the broken line in (a). You can see even larger stress is localized at the corners in Fig. 9 (a). The stress is caused by the densification of glass during the processing. It is noted that the stress and index change are released by annealing and strongly coupled to each other. It seems possible to control the amount of index change by changing the stress by some means.
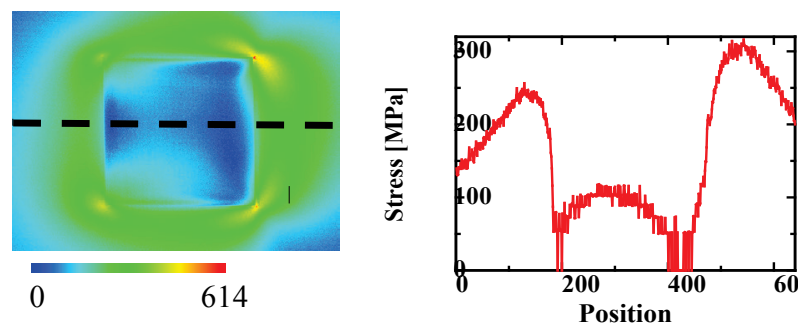

Principal stress difference [MPa]

Fig. 9. Stress distribution in processed glass.

Eaton et all reported a successful fabrication experiment of asymmetric waveguide structures with high refractive index $\left(2.2 \times 10^{-2}\right)$ using high-repetition- rate laser pulses $(500 \mathrm{KHz})$ [22]. Hashimoto at al demonstrated just recently that even low-repetition-rate $(1 \mathrm{KHz})$ laser pulses can induce high refractive index changes [23]. They observed a movement of void during the fabrication process and reasoned that the movement of void contributes to the index enhancement and the formation of asymmetric cross-section of the waveguide. The author believes that the generation and movement of void relaxes the strong tensile stress in the glass network and helps the compaction or densification of processed material.

\section{Future and Challenges}

Ultrafast laser microprocessing is a kind of additive and $3 \mathrm{D}$ fabrication technique like the $3 \mathrm{D}$ printers. It is also simple like 3D printers and allows processing in air and room temperature. Active devices like light sources, amplifiers or detectors can be included into a total system by joining the glass and silicon or other semiconductor subsystems. A part of glass system can also be doped with active materials. If necessary, holes can be made [24-26] for flow channels, power lines or areas of active materials. Highly integrated systems can be expected in the near future.

The present technique provides the way of abovementioned joining technique such as the wafer bonding and that of hermetic sealing for delicate devices such as semiconductor sensors, MEMS and solar cells where room-temperature processing is mandatory. Welding 
techniques that join transparent materials without absorbing or adhesive layers are widely desired and especially for bio fluidics.

For future applications, fast and wide-area welding technique is of critical importance. The techniques of burst pulses [27, 28] or double pulses [29] enhance the efficiency of laser welding and also provide the high controllability. The novel idea of the prebonding drastically improved the joining strength and reliability of ultrafast laser microwelding [30, 31]. Miyamoto further clarified the crack-free condition that is related to the dual-structure of the processed area $[32,33]$. A new idea such as the reinforced direct bonding [34] is attractive for fast and wide welding. We may expect and need further more the advent of novel techniques for practical applications.

Another issue may be a laser system. We need high-power and high rep.-rate and possibly a compact laser system with reasonable price for enhancing the practical applications. The semiconductor laser coupled with a fiber amplifier may be the most promising industrial light sources for the near future. I believe that the possible instability of such new laser systems may be compensated by electronic feedback systems in the fabrication process.

\section{Conclusion}

After brief introduction of filamentation in glass, the filament-based fabrication techniques for photonic devices such as the waveguide, coupler, Bragg grating mirror, and diffractive lens were briefly reviewed and laser welding techniques for glass-glass and glass-metal joining were introduced including the application to the hermetic sealing. Then, the author gave an idea of the relationship between the void formation, residual stress, and index change and secure welding. Finally the future challenges to the fast, wide and stable welding techniques were touched on.

\section{Acknowledgment}

The author would like to express sincere thanks to Profs. W. Watanabe, T. Tamaki, and Y. Ozeki for their support during the research works.

\section{References}

[1] E. N. Glezer et al., Opt. Lett. 21, (1996) 2023.

[2] W. Watanabe et al., Opt. Lett. 25, (2000) 1669.

[3] K. M. Davis et al., Opt. Lett. 21, (1996) 1729.

[4] T. Tamaki et al., Jpn. J. Appl. Phys. 44, (2005) L687.

[5] K. Itoh et al., MRS Bulletin 31, (2006), 620.

[6] R. R. Gattass and E. Mazur, Nature Photonics 2, (2008) 219.

[7] K. Itoh, W. Watanabe and Y. Ozeki, Proc. IEEE 97, (2009) 1011.

[8] W. Watanabe et al., in Progress in Ultrafast Intense Laser Science VI, Springer Series in Chemical Physics 99, (2010) 161.

[9] A. Couairon and A. Mysyrowicz, Physics Reports - Review Section of Physics Letters 441, (2007) 47.

[10] L. Berge et al, Reports on Progress in Physics 70, (2007) 1633.

[11] K. Yamada et al., J. Appl. Phys. 42, (2003) 6916.

[12] W. Watanabe et al., Opt. Lett. 28, (2003) 2491.

[13] K. Yamada et al., Opt. Lett. 29, (2004)1846.

[14] S.M. Eaton et al, Opt. Express 13, (2005) 4708.
[15] W. Watanabe et al., Appl. Phys. Lett. 89, (2006) 021106.

[16] Y. Ozeki et al., Appl. Phys. Express 1, (2008) 082601.

[17] Y. Ozeki et al., Proc. LAMP 2009, (2009) 09-115.

[18] T. Yoshino et al, Jpn. J. Appl. Phys., 51, (2012) 102403.

[19] F. Hashimoto et al, Proceedings of LAMP2013 the 6th International Congress on Laser Advanced Materials Processing, \#13-018:A176 (2013).

[20] J.W. Chan et al., Appl. Phys., A 76, (2003) 367.

[21] K. Itoh and Y. Ozeki, in 2010 ICALEO Conference Proceedings, M1001(2010).

[22] S.M. Eaton et al., J. Non-Cryst. Solids 357, (2010) 2387.

[23] K. Hashimoto et al, Jap. J. Appl. Phys. 53, (2014) 042601.

[24] Y. Kondo et al, Jpn. J. Appl. Phys. 38, (1999) L1146.

[25] J. Wang et al, Appl. Phys. A 68, (1999) 111.

[26] Y. Li et al, Opt. Lett. 26, (2001) 1912.

[27] P.R. Herman et al., US Patent 6552301 B2, (2003).

[28] F. Zimmermann et al., Appl. Opt. 52 (2013) 1149.

[29] K. Sugioka et al., Opt. Lett.36,(2011)2734.

[30] K. Cvecek et al., Appl. Opt. 50, (2011), 1941.

[31] I. Miyamoto et al., Opt. Express 19, (2011) 22961.

[32] I. Miyamoto, K. Cvecek, M. Schmidt, Opt. Express 21, (2013) 14291.

[33] I. Miyamoto et al., Appl. Phys. A 114, (2014)187.

[34] D. Hélie et al, Appl. Opt. 51 (2012) 2098.

(Received: June 13, 2014, Accepted: September 26, 2014) 